\title{
Effect of main belt perturbers on asteroid-pair age estimation
}

\author{
A. Galád ${ }^{1,2}$ \\ ${ }^{1}$ Dept. of Astronomy, Physics of the Earth, and Meteorology, FMFI, Comenius University, 84248 Bratislava, Slovakia \\ e-mail: galad@fmph.uniba.sk \\ 2 Astronomical Institute, AS CR, 25165 Ondřejov, Czech Republic
}

Received 20 June 2012 / Accepted 6 September 2012

\begin{abstract}
Context. Asteroid-pair age estimations are usually performed by backward integration of possible orbits of pair components, taking planetary perturbations into account along with the Yarkovsky effect. It is assumed that uncertainties coming from the backward integration process itself are small, so to reduce uncertainties in estimations, effort usually focuses on reducing uncertainties in the initial orbital elements and the dimensions of the pair components.

Aims. This work aims to evaluate the role of the frequently ignored nonplanetary perturbers in asteroid-pair age estimation. When their role is not negligible, the ages of the youngest known pairs can be roughly re-estimated.

Methods. The orbital evolution of several asteroid-pair components and the close approaches between components during the last $\approx 43 \mathrm{kyr}$ are investigated. Initially, the force equations consisted of only planetary perturbers. The three largest main belt perturbers were added afterwards. Finally, as many as 262 massive main belt perturbers were included. The effect of main belters on age estimation is assessed by comparing the dates of the closest approaches between pair components. The range of the Yarkovsky effect is simulated very roughly, only for comparison purposes.

Results. The estimated age of the youngest known pair (6070) Rheinland - (54827) $2001 \mathrm{NQ}_{8}$ including the Yarkovsky effect seems to be either $16.3 \pm 0.1 \mathrm{kyr}$ for the retrograde rotation or $16.0 \pm 0.2 \mathrm{kyr}$ for the prograde rotation of the second component, respectively. This is younger by about $0.7-0.9 \mathrm{kyr}$ than the previous estimate. Several more pairs, namely (88259) $2001 \mathrm{HJ}_{7}-1999 \mathrm{VA}_{117}$, (111962) $2002 \mathrm{GP}_{75}$ - (280008) $2001 \mathrm{UR}_{224}$, (180906) $2005 \mathrm{~KB}_{6}-(217266) 2003 \mathrm{YR}_{67}$, (229401) $2005 \mathrm{SU}_{152}-2005 \mathrm{UY}_{97}$, and $2005 \mathrm{GS}_{180}-2008 \mathrm{FK}_{107}$, had relative encounter velocities within $1.0 \mathrm{~m} \mathrm{~s}^{-1}$, suggesting they might also have formed within the interval studied. Some other pairs, including (5026) Martes - $2005 \mathrm{WW}_{113}$, which was previously reported as very young, had slightly higher encounter velocities and longer nominal minimum distances, calling into question whether they could have formed within the interval studied or in some previous mutual encounter of pair components.

Conclusions. The effect of main belt perturbers on asteroid-pair age estimation is buried in the huge age uncertainties caused by the Yarkovsky effect until the rotation direction of pair components is recognized.
\end{abstract}

Key words. minor planets, asteroids: general - celestial mechanics

\section{Introduction}

Asteroid pairs or clusters are small groups of bodies that share almost the same heliocentric orbit. Unlike so-called multiple asteroid systems, the members are no longer bound together gravitationally, but backward integration of their individual orbits suggests that they had a common origin. It can also indicate when they might have been sufficiently close to have been under each other's gravitational influence (within the Hill sphere). Dozens of such asteroid pairs have been found (Vokrouhlický \& Nesvorný 2008; Pravec \& Vokrouhlický 2009; Pravec et al. 2010), as have a few young clusters (e.g. Nesvorný et al. 2002, 2003, 2006; Nesvorný \& Bottke 2004; Nesvorný \& Vokrouhlický 2006).

Members of a given asteroid pair/cluster have similar values for three proper elements, as well as the five Keplerian orbital elements other than the mean anomaly. The degree of their orbital similarity depends on their location in the solar system, the age of the pair/cluster, and even the size of the component bodies. Given the dimensions of known pair/cluster components (typically $\approx 1-10 \mathrm{~km}$ ), the Yarkovsky effect is important for their orbit evolution. This effect has been confirmed and measured in some
near-Earth asteroids (e.g. Chesley et al. 2003; Vokrouhlický et al. 2008). The drift it causes in semimajor axes is tiny on a short time scale, but has a major impact over the much longer periods required for age estimation of pairs/clusters.

Considering the uncertainties in the initial orbital elements and in modeling parameters like the Yarkovsky effect, one can appreciate the difficulty of computing possible orbit intersections $10^{5}-10^{6} \mathrm{yr}$ ago with sufficient precision/accuracy to make age estimations. Although the uncertainties in the initial orbital elements are relatively small, there are usually much larger uncertainties in modeling the Yarkovsky effect, which depends not only on distance from the Sun, but also on many physical parameters of individual asteroids, such as size, surface properties, shape, obliquity, and rotation rate (e.g. Bottke et al. 2002). The effect of these uncertainties grows with time during integration, and long-term backward integration (e.g. $>10^{6} \mathrm{yr}$ ) is very time-consuming.

In studies of this sort, one usually integrates orbits for a set of bodies representing each asteroid in the pair/cluster. In addition to a nominal orbit based on the initial orbital elements, there are variant orbits based on slightly different starting conditions that are within the uncertainties of the initial elements 
Table 1. Basic selection criteria for orbits of asteroid-pair component candidates.

\begin{tabular}{llll}
\hline \hline & Proper & & Orbital \\
\hline$\Delta a_{\mathrm{p}}$ & $\leq 0.0005 \mathrm{AU}$ & $\Delta a$ & $\leq a_{\mathrm{p}} \times 0.0021 \mathrm{AU}$ \\
$\Delta e_{\mathrm{p}}$ & $\leq 0.0005$ & $\Delta e$ & $\leq 0.0042$ \\
$\Delta \sin i_{\mathrm{p}}$ & $\leq 0.0005$ & $\Delta i$ & $\leq 0.03^{\circ}$ \\
& & $\Delta \Omega$ & $<1.5^{\circ}$ \\
& & $\Delta \omega$ & $<3.0^{\circ}$ \\
\hline
\end{tabular}

(geometrical clones). Other variant orbits might be computed using different values of inadequately known modeling parameters, like the Yarkovsky effect (Yarkovsky clones).

Additional astrometric observations, recent or ancient, help improve the initial orbital elements and reduce their uncertainty. Photometric observations help solidify the estimate of absolute magnitude $H$, from which a body's dimension can be deduced, assuming albedo is estimated, and the light curves from several apparitions help derive the sense of rotation. The $H$ value and sense of rotation heavily reduced uncertainties in age estimation in the case of the very young pair (6070) Rheinland (54827) $2001 \mathrm{NQ}_{8}$ (Vokrouhlický et al. 2011). Two other very young pairs are known, namely (5026) Martes - $2005 \mathrm{WW}_{113}$ and (229401) $2005 \mathrm{SU}_{15}-2005 \mathrm{UY}_{97}$, which seem to have a similar age of $\sim 17 \mathrm{kyr}$ (Pravec et al. 2010). At the present time there are no younger pairs or clusters reported in the literature.

Trial computations that include selected main belt perturbers (at least Ceres) have suggested that their role in estimating the age of young clusters/families (usually around $10^{5}-10^{6} \mathrm{yr}$ ) might be minor in comparison to the role of the Yarkovsky effect. According to Laskar et al. (2011), the long term (over $400 \mathrm{kyr}$ ) motion of Ceres and Vesta will be unpredictable even after precise position measurements are available from space missions. Moreover, close encounters with such large perturbers produce strong chaotic behavior in orbits of other asteroids. It is no wonder then that to simplify and speed up the integration process, main belt perturbers are often ignored.

Nonetheless, since some asteroid pairs are quite young $\left(10^{4}-10^{5} \mathrm{yr}\right)$ and may have undergone repeated close approaches to massive main belters, this work aims to account for a few such nonplanetary perturbers and to independently estimate the age of the youngest known asteroid pairs.

\section{Finding young asteroid pairs}

The first step in finding young asteroid pairs or clusters was to establish measures of similarity for orbits with which to examine databases of proper $^{1}$ and Keplerian elements ${ }^{2}$. For a given epoch, these four different Keplerian element databases provide nearly identical data for numbered and multiopposition objects. Since the single opposition objects were ignored in this study, any of the four could be used at this stage.

Criteria for this similarity in elements were chosen somewhat empirically after observing the variation in osculating orbital elements over the time span of $30 \mathrm{kyr}$ for a few known pairs. Information about the integrator used for this task is given in the paragraph below. The basic criteria are listed in Table 1 , where $a$ is the semimajor axis, $e$ the eccentricity, $i$ the inclination, $\Omega$ the

\footnotetext{
1 http://hamilton.dm.unipi .it/astdys2/index .php?pc=5

2 J: http://ssd.jpl.nasa.gov/?sb_elem

B: ftp://ftp. lowell.edu/pub/elgb/astorb.html

A: http://hamilton.dm . unipi . it/astdys $2 /$ index $\cdot$ php?pc $=4$

M: http://www . minorplanetcenter .net/iau/MPCORB .html
}

Table 2. Approximate dates of recent close and slow approaches between components of asteroid pairs computed without/with CPV perturbers.

\begin{tabular}{rrrrr}
\hline \hline \multicolumn{2}{c}{ Asteroid pair } & $\begin{array}{c}t_{0} \\
{[\mathrm{kyr} \text { B.C. }]}\end{array}$ & $\begin{array}{c}t_{\mathrm{CPV}} \\
{[\mathrm{kyr} \text { B.C. }]}\end{array}$ & $\begin{array}{c}a_{\mathrm{p}} \\
{[\mathrm{AU}]}\end{array}$ \\
\hline $2384-$ & $2009 \mathrm{EL}_{11}$ & $40.9-39.8$ & $>41.0-40.6$ & 2.61 \\
$5026-2005 \mathrm{WW}_{113}$ & $15.7-14.9$ & $15.8-15.0$ & 2.38 \\
$6070-$ & 54827 & $15.3-15.2$ & 14.2 & 2.39 \\
$8898-$ & 70287 & $9.4-9.3$ & 9.4 & 2.43 \\
$39991-$ & $2008 \mathrm{YV}_{80}$ & 5.3 & 4.9 & 2.44 \\
$70335-$ & $2002 \mathrm{PX}_{197}$ & $8.7-8.6$ & $9.1-9.0$ & 2.44 \\
$80218-$ & 213471 & 11.3 & 11.3 & 2.22 \\
$88259-$ & $1999 \mathrm{VA}_{117}$ & $30.5-30.4$ & $30.8-30.5$ & 1.94 \\
$99052-$ & 291788 & 3.2 & 3.2 & 2.73 \\
$100440-$ & $2011 \mathrm{SE}_{164}$ & $(32.9-26.3)$ & $(36.6-27.4)$ & 2.34 \\
$111962-$ & 280008 & $39.6-39.5$ & $38.5-37.8$ & 2.34 \\
$125887-$ & 197706 & 9.1 & 9.1 & 2.40 \\
$180906-$ & 217266 & $19.8-19.7$ & $>41.0-33.9$ & 2.24 \\
$224801-$ & $2008 \mathrm{SZ}_{124}$ & $13.4-13.3$ & $13.8-12.8$ & 2.15 \\
& & $37.1-37.0$ & $38.7-35.2$ & \\
$229401-$ & $2005 \mathrm{UY}_{97}$ & $>41.0$ & $16.3-15.1$ & 2.64 \\
$268305-$ & 282920 & 24.9 & $25.4-24.7$ & 2.39 \\
$2005 \mathrm{GS}_{180}-$ & $2008 \mathrm{FK}_{107}$ & $>41.0$ & $41.3-34.3$ & 2.44 \\
\hline \multicolumn{7}{r}{} & & & &
\end{tabular}

longitude of node, and $\omega$ the argument of perihelion; the subscript $\mathrm{p}$ indicates proper elements. Additional criteria had to be chosen for nearly circular orbits $(e \ll)$ and for $\Omega / \omega$ approaching $0^{\circ} / 360^{\circ}$. Use of these criteria revealed more than 200 candidates for young asteroid pairs (and a few clusters), most of which had been identified in previous studies. Known pairs that did not meet these criteria are probably much older than $30 \mathrm{kyr}$ or outside the main belt. For example, the pair (9068) 1993 OD - 2002 $\mathrm{OP}_{28}$ consists of Mars-crossers for which proper elements were unavailable, though its age was estimated to be $\approx 32 \mathrm{kyr}$ (Pravec et al. 2010).

The second step in the discovery process is backward integration, where we used the SOLEX package (Vitagliano 1997) ${ }^{3}$. This numerically integrates the solar system, producing planetary ephemerides that closely match JPL's DE406 or DE421 and can incorporate numbered asteroids by reading the elements from AstDys or Astorb databases. It has the option of including or ignoring perturbations by the three largest main belters (Ceres, Pallas, Vesta, hereafter referred to as CPV). In addition, users can input their own lists of orbital elements, even for other objects (e.g. unnumbered asteroids), and specify masses of the added bodies.

Table 2 lists the approximate dates (in kyr B.C.) of approaches in the last $43 \mathrm{kyr}, t_{0}$ is for computations with just planets as perturbers, and $t_{\mathrm{CPV}}$ for computations taking also CPV as perturbers into account. A pair is included if the encounter velocity (at infinity) of its components is $\leq 5.0 \mathrm{~m} \mathrm{~s}^{-1}$ and the nominal closest approach is within $10^{5} \mathrm{~km}$. Rough values for asteroids' proper semimajor axes $a_{\mathrm{p}}$ are listed for comparison purposes; by comparison, for CPV the values are 2.77, 2.77, and 2.36 AU. The encounter dates for asteroid pair (100440) $1996 \mathrm{PJ}_{6}-2011 \mathrm{SE}_{164}$ are in parentheses because they were not found using two databases. Actually, (2384) Schulhof with 2009 $\mathrm{EL}_{11}$ and (39991) $1998 \mathrm{HR}_{37}$ with $2008 \mathrm{YV}_{80}$ are members of clusters, but they are also referred to as pair components from here on.

\footnotetext{
Updated version at

http://chemistry. unina.it/ alvitagl/solex/
} 
Looking at results going back $\approx 30 \mathrm{kyr}$, for many pairs, the approximate dates of recent close and slow approaches of their components were quite similar regardless of whether CPV were included or excluded, regardless of which JPL ephemeris was chosen, and regardless of which database provided the initial conditions. But for a few pairs, there were considerable differences between results with and without CPV. On a longer time span, slight differences were seen, also depending on the chosen ephemeris and database. JPL and MPC databases provided the same results as the orbital elements of the used asteroids were the same.

Most of the dates in Table 2 should not be considered as those of pair formation because members of any pair can encounter each other repeatedly (typically three times within $100 \mathrm{kyr}$ ). Neither the Yarkovsky effect nor main belt perturbers other than CPV were included in these computations. Also the escape velocity $v_{\mathrm{esc}}$ for most of the listed components is much lower than $5.0 \mathrm{~m} \mathrm{~s}^{-1}$. According to

$v_{\mathrm{esc}}=D \sqrt{\frac{2 \pi \rho G}{3}}$,

where $D$ is the effective diameter of the asteroid, $\rho$ its bulk density, $G$ the universal constant of gravitation, bodies with effective diameter exceeding $\approx 9.5 \mathrm{~km}$ have an escape velocity that exceeds $5.0 \mathrm{~m} \mathrm{~s}^{-1}$, assuming bulk density $2000 \mathrm{~kg} \mathrm{~m}^{-3}$. Among the listed asteroids, only (2384) Schulhof is likely to be that large. Sizes are indirectly estimated from the absolute magnitudes $H$, which themselves are not precisely known, and from the assumed geometrical albedos. The $H$ value for (2384) Schulhof is 11.7-12.5 according to the database used. Assuming an albedo of 0.183 , the effective diameter would exceed $9.8 \mathrm{~km}$ (see also Nathues 2010; Vokrouhlický \& Nesvorný 2011). Nevertheless, its Hill radius

$R_{\mathrm{Hill}} \approx a \sqrt[3]{\frac{m}{3 M}}$

where $a$ is the semimajor axis, $m$ the mass of the asteroid, and $M$ the solar mass, is only $\approx 2-3 \times 10^{3} \mathrm{~km}$.

\section{Procedure}

To investigate the impact of bodies in addition to CPV on asteroid-pair age estimation, the threshold was set at $1 / 1000$ mass of (1) Ceres, i.e. $>\approx 0.9 \times 10^{18} \mathrm{~kg}$, so 262 main belt bodies (including CPV) were selected with assumed masses higher than that. A few potential perturbers close to the limit might have been omitted unwittingly, because the masses of such asteroids were usually only estimated from effective diameters (e.g. Tedesco et al. 2002; Usui et al. 2011), and/or from absolute magnitude $H$ and their supposed geometrical albedo, using an assumed bulk density of $2000 \mathrm{~kg} \mathrm{~m}^{-3}$. But their role should not be as critical for this study as is the role of their larger counterparts. Masses of the larger ones have been directly derived from astrometry (e.g. Baer et al. 2011; Zielenbach 2011 and references therein). In fact, the criterion mentioned above implies an effective diameter $D>\approx 90 \mathrm{~km}$. About 25 additional asteroids located in the Trojan region could also pass the criteria of mass or diameter, but their perturbations on the pair members located in the main belt can be ignored.

In addition to the four previously mentioned Keplerian element databases, one more database was constructed using the FIND_ORB software developed by B. Gray ${ }^{4}$. This new database

\footnotetext{
4 http://www.projectpluto.com/find_orb.htm
}

was intended to contain improved orbital elements especially for the perturbers. The improvement would come by limiting the observations used for differential correction of the orbits to only the more recent ones. It was felt that orbital elements uncertainties could be lower with a shorter set of data than when observations from the entire observation arc were processed without perturbations from additional large main belters other than CPV. With FIND_ORB, one can choose the perturbers, the number of decimal places for derived orbital elements, the epoch for those osculating elements, and also the rejection limits for astrometric residuals to be used in determining the orbit. All available perturbers were chosen (planets, as well as the three largest main belters, i.e. CPV), which is standard in all other databases. The limit for residuals was set to $2.0^{\prime \prime}$, which is also comparable to other databases. The number of decimal places was enhanced by two in comparison to the JPL, Astorb, and MPC databases. It was only a formal change, since the uncertainties in elements were usually at their last decimal places in those databases. Unlike the other databases, only observations between January 1991 and February 2012 were used, and the chosen epoch of osculation was 2004 July 14, well within the interval covered by observations (in contrast to the epoch 2012 March 14 used in other databases). Only a few unnumbered members of pairs lacked astrometric observations prior to 2004 .

With the starting conditions from each of the five databases, SOLEX integrated all pair components listed in Table 2 backward $\approx 43 \mathrm{kyr}$. In special cases the interval was increased slightly. The planetary positions came from JPL's DE406, and the force equations also included the 262 mainbelt perturbers mentioned earlier. In fact, equations of motion are not available to the user. SOLEX's input file contains either orbital elements or positions with velocities for individual asteroids. Among output files, there are two files having such a structure so that they could be directly used as another input so that computations may continue. But output files can also be edited to prepare different input according to user's wishes. Although the current version of SOLEX does not have a direct option for modeling a continuous drift in the semimajor axis yet, the Yarkovsky effect could be roughly simulated by periodic adjustment of the semimajor axis of each member of pairs by a fixed amount. To this end, two additional backward integrations were run for each set of starting conditions. The first added a specific value (every $-2000 \mathrm{yr}$ starting from year 1000) to the semimajor axis (representing retrograde rotations of pair components), and the second repeatedly subtracted that amount from the semimajor axis (prograde rotations). The sizes of these shifts were chosen roughly according to absolute magnitudes, from the four values of $7.5,11.25,15.0$, and $22.5 \mathrm{~m} \mathrm{yr}^{-1}$ (i.e., every $-2000 \mathrm{yr}$ the drift in $a$ was $+/-1.0,1.5,2.0$, and $3.0 \times 10^{-7} \mathrm{AU}$, respectively). The values were meant to be typical. Maximum possible values may be a bit higher and, for the smallest asteroids located in the inner part of the main belt the values, perhaps a few times higher (e.g. Bottke et al. 2006; Vokrouhlický \& Nesvorný 2009; Duddy et al. 2012).

\section{Results and discussion}

The main results of backward integrations using starting conditions from the five orbital element databases - AstDys (A), Astorb (B), Find_ORB (F), JPL (J), MPC (M) - are summarized in Table 3 with nominal dates $t$, minimum distances $d$, and encounter velocities $v$ of recent approaches between the components of asteroid pairs. The last column contains nominal 
Table 3. Nominal dates $t$, distances $d$, and encounter velocities $v$ of recent approaches between components of asteroid pairs computed with 262 main belt perturbers (strongest ones are listed) using different orbital element databases ELM.

\begin{tabular}{|c|c|c|c|c|c|c|c|c|c|}
\hline \multicolumn{2}{|c|}{$\begin{array}{l}\text { Asteroid pair and } \\
\text { strongest perturbers }\end{array}$} & & ELM & $\begin{array}{c}d \\
{\left[10^{3} \mathrm{~km}\right]}\end{array}$ & $\begin{array}{c}v \\
{\left[\mathrm{~m} \mathrm{~s}^{-1}\right]}\end{array}$ & $\begin{array}{l}t \\
{[\mathrm{yr}]}\end{array}$ & $\begin{array}{c}t_{\mathrm{p}} \\
{[\mathrm{yr}]}\end{array}$ & $\begin{array}{c}t_{\mathrm{r}} \\
{[\mathrm{yr}]}\end{array}$ & $\begin{array}{l}t_{\mathrm{CPV}} \\
{[\mathrm{yr}]}\end{array}$ \\
\hline \multirow{2}{*}{\multicolumn{3}{|c|}{ 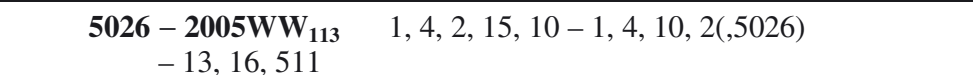 }} & & & \multicolumn{5}{|c|}{$\Delta a / \Delta t= \pm 7.5- \pm 22.5 \mathrm{~m} \mathrm{yr}^{-1}$} \\
\hline & & & A & 40 & 1.1 & -15246 & -15222 & -15253 & -15237 \\
\hline 29,145 & \multicolumn{2}{|l|}{$-15,324$} & B & 62 & 2.2 & -14956 & -14948 & -15000 & -14965 \\
\hline 19 & \multicolumn{2}{|l|}{-15} & $\mathrm{~F}$ & 76 & 1.8 & -14981 & -14943 & -14995 & -14965 \\
\hline 29 & \multicolumn{2}{|c|}{$-88,15,776,511$} & $\mathrm{~J}$ & 17 & 0.6 & -15826 & -15770 & -15862 & -15822 \\
\hline 29,145 & \multicolumn{2}{|c|}{$-15,776,511,88$} & M & 92 & 3.7 & -15824 & -15764 & -15863 & -15822 \\
\hline \multicolumn{2}{|c|}{$6070-\quad 54827$} & \multicolumn{3}{|l|}{$1,4,9,6,19,308,29,324,3-1,4,6,19,10,9,8$} & \multicolumn{5}{|c|}{ $\pm 7.5- \pm 15.0 \mathrm{~m} \mathrm{yr}^{-1}$} \\
\hline \multirow[t]{2}{*}{45,144} & \multicolumn{2}{|l|}{-} & $\mathrm{A}$ & 3 & 0.3 & -14103 & -13939 & -14272 & -14202 \\
\hline & \multirow{2}{*}{\multicolumn{2}{|c|}{-}} & B & 11 & 0.5 & -14242 & -13967 & -14222 & -14212 \\
\hline & -128 & & $\mathrm{~F}$ & 12 & 0.4 & -14231 & -13964 & -14220 & -14214 \\
\hline \multirow[t]{2}{*}{45} & \multicolumn{2}{|l|}{-} & $\mathrm{J}$ & 12 & 0.5 & -14198 & -13953 & -14279 & -14227 \\
\hline & - & & $\mathrm{M}$ & 11 & 0.5 & -14209 & -13924 & -14324 & -14227 \\
\hline \multicolumn{3}{|c|}{$80218-\quad 213471$} & & & & \pm 1 & $.0- \pm 15$ & $0 \mathrm{~m} \mathrm{yr}^{-1}$ & \\
\hline 21,139 & - & & A & 34 & 1.6 & -11401 & -11303 & -11449 & -11331 \\
\hline & - & & $\mathrm{B}$ & 34 & 1.5 & -11404 & -11309 & -11463 & -11334 \\
\hline 7 & - & & $\mathrm{F}$ & 37 & 2.0 & -11403 & -11308 & -11469 & -11335 \\
\hline 7 & - & & $\mathrm{J}$ & 31 & 2.2 & -11400 & -11302 & -11434 & -11331 \\
\hline 7 & - & & M & 31 & 2.2 & -11400 & -11305 & -11425 & -11331 \\
\hline 88 & $9-1999 V_{117}$ & $2,3-2$ & & & & \pm 11 & $25- \pm 22$ & $.5 \mathrm{~m} \mathrm{yr}^{-1}$ & \\
\hline & - & & A & 2 & 0.1 & -30497 & -29583 & -32452 & -30523 \\
\hline 41 & -7 & & $\mathrm{~B}$ & 3 & 0.1 & -30773 & -29572 & -32577 & -30531 \\
\hline & -7 & & $\mathrm{~F}$ & 2 & 0.1 & -30731 & -29521 & -32527 & -30504 \\
\hline 41 & - & & $\mathrm{J}$ & 3 & 0.1 & -30741 & -29574 & -32676 & -30708 \\
\hline & -7 & & M & 3 & 0.1 & -30771 & -29571 & -32604 & -30708 \\
\hline 111 & $2-\quad 280008$ & $1,4,2,15,19,3,10-1,4,2,10$ & & & & & $0- \pm 22$ & $.5 \mathrm{~m} \mathrm{yr}^{-1}$ & \\
\hline 21,11 & $-19,5,3,14$ & & A & 16 & 0.7 & -38890 & -39635 & -39420 & -38543 \\
\hline 128,55 & $15-15,5,16,2$ & 11 & $\mathrm{~B}$ & 18 & 09 & -38669 & -42584 & -39316 & -38514 \\
\hline 45,128 , & $-15,45,5,2$ & $16,511,29,324$ & $\mathrm{~F}$ & 18 & 0.9 & -38762 & -43062 & -39289 & -38486 \\
\hline 13,409 & $92,769,11-15$ & $9,3,324,20,511,240,29,128$ & $\mathrm{~J}$ & 15 & 0.7 & -38640 & -40602 & -39359 & -38479 \\
\hline 45,11 & $128-15,3,5$ & $19,29,511$ & M & 17 & 0.9 & -38776 & -43024 & -39269 & -38479 \\
\hline 180 & $6-217266$ & $4,1,2,15,13,88-4,1,2,15,13$ & & & & & $2.5- \pm 22$ & $.5 \mathrm{~m} \mathrm{yr}^{-1}$ & \\
\hline $3,14,2$ & $40-8,88,3,2$ & & A & 2 & 0.1 & -31906 & -31273 & -34301 & -35883 \\
\hline $3,19,5$ & $29-54,40,9$ & & $\mathrm{~B}$ & & & $<-45000$ & -43361 & ? & -33866 \\
\hline 3,747 & $40,29-88,8,3$ & & $\mathrm{~F}$ & 3 & 0.1 & -36696 & -45072 & $?$ & -34092 \\
\hline 9,521 & $29-54,20$ & & $\mathrm{~J}$ & 2 & 0.1 & -37521 & -40016 & $?$ & -36470 \\
\hline $3,7,19$ & $30,521,9-140$ & $9,54,20,3,9$ & M & 3 & 0.1 & -42442 & -40523 & ? & -36470 \\
\hline 224 & $1-2008 S Z_{124}$ & $4,1,2,19,3,18\left(, 2008 \mathrm{SZ}_{124}\right)-4,1,2,19,9,3$ & $7(, 224$ & 1801) & & \pm 2 & $2.5- \pm 22$ & $.5 \mathrm{~m} \mathrm{yr}^{-1}$ & \\
\hline $21,9,6$ & $40-5,18,20,3$ & & A & 55 & 2.8 & -35678 & -37132 & -36273 & -35230 \\
\hline 9,345 & $-6,20,21,5$ & 324 & B & 10 & 1.0 & -36833 & -37502 & -38913 & -37183 \\
\hline 9,30 & $-8,51,20,6$ & & $\mathrm{~F}$ & 47 & 2.1 & -36804 & -37321 & -38791 & -37174 \\
\hline 6 & $-6,18,21$ & & $\mathrm{~J}$ & 39 & 1.7 & -38345 & -37117 & -36342 & -38654 \\
\hline $9,6,30$ & $1-6,18$ & & M & 54 & 2.7 & -38349 & -36930 & -36351 & -38654 \\
\hline 229 & $1-2005 U Y_{97}$ & $1,4,10,3-1,4,10,2,704$ & & & & & $.0- \pm 22$ & $.5 \mathrm{~m} \mathrm{yr}^{-1}$ & \\
\hline & $-451,13$ & & A & 1 & 0 & -15803 & -25929 & -14197 & -15425 \\
\hline 196 & -13 & & B & 2 & & -15691 & -24178 & -13577 & -15120 \\
\hline 196 & -13 & & $\mathrm{~F}$ & 2 & 0 & -15640 & -25882 & -13517 & -15098 \\
\hline 196 & -16 & & $\mathrm{~J}$ & 3 & 0 & -16426 & -26028 & -15313 & -16288 \\
\hline 196 & -16 & & M & 1 & 0.2 & -16467 & -25908 & -15347 & -16288 \\
\hline 2005GS & $-2008 \mathrm{FK}_{107}$ & $4,2,10,511,704,16,29,19,24,88-1,4,10$ & 2,704, & 16,88 & & & $.5- \pm 22$ & $.5 \mathrm{~m} \mathrm{yr}^{-1}$ & \\
\hline 14,275 , & $9,27,52,128$ & $2-9,14,8,11,24,29,42,45,51,54,128,171$ & A & 9 & 0.3 & -43518 & -32639 & -42561 & -34344 \\
\hline 14,27 & $6,128-9,52,1$ & $7,8,19,139,511$ & $\mathrm{~B}$ & 9 & 0.3 & -32606 & -32864 & -37821 & -36394 \\
\hline 106,14 & $-511,19,13$ & $8,7,52$ & $\mathrm{~F}$ & 11 & 0.3 & -35596 & -32179 & -37997 & -36388 \\
\hline 9,106 & $5-16,51,139$ & $5,7,8,13,19,52$ & $\mathrm{~J}$ & 6 & 0.1 & -34183 & -31888 & -37725 & -36431 \\
\hline 14,106 , & $94-13,15,139$ & $50,19,7$ & M & 9 & 0.2 & -31558 & -37974 & -37712 & -36431 \\
\hline
\end{tabular}

Notes. $t_{\mathrm{p}}$ and $t_{\mathrm{r}}$ are nominal dates of approaches for prograde and retrograde rotators, respectively.

dates $t_{\mathrm{CPV}}$ with the simple inclusion of $\mathrm{CPV}^{5}$. The difference between $t$ and $t_{\mathrm{CPV}}$ values provides a rough idea of how important the additional perturbers (other than CPV) are.

\footnotetext{
5 Table 2 also contains these values, but only approximately, mixed for all databases and for both ephemerides.
}

Along with the distances and velocities of approaching objects with given masses, SOLEX also computes several parameters characterizing encounters, such as deflection angle, relative change in semimajor axis, or the mean motion variation undergone by the lighter body. The strongest main belt perturbers that 
Table 4. Nominal dates of approaches between a few Yarkovsky clones of asteroids (6070) Rheinland and (54827) $2001 \mathrm{NQ}_{8}$ computed with 262 main belt perturbers using the $\mathrm{F}$ database.

\begin{tabular}{llllllllllll}
\hline \hline $\mathrm{m} \mathrm{yr}^{-1}$ & -30.0 & -15.0 & -9.0 & -6.0 & -3.0 & $\begin{array}{c}(54827) \\
{[\mathrm{yr}]}\end{array}$ & +3.0 & +6.0 & +9.0 & +15.0 & +30.0 \\
\hline-15.0 & -14428 & -14054 & -14041 & -14028 & -14009 & -13980 & -13923 & -13829 & -13773 & -13686 & -13478 \\
-9.0 & -14578 & -14194 & -14180 & -14167 & -14147 & -14119 & -14058 & -13962 & -13903 & -13816 & -13601 \\
-7.5 & -14606 & $\mathbf{- 1 4 2 2 0}$ & $\mathbf{- 1 4 2 0 5}$ & $\mathbf{- 1 4 1 9 1}$ & $\mathbf{- 1 4 1 7 2}$ & -14143 & $\mathbf{- 1 4 0 8 3}$ & $\mathbf{- 1 3 9 8 6}$ & $\mathbf{- 1 3 9 2 7}$ & $\mathbf{- 1 3 8 3 7}$ & -13623 \\
-6.0 & -14641 & $\mathbf{- 1 4 2 5 0}$ & $\mathbf{- 1 4 2 3 5}$ & $\mathbf{- 1 4 2 2 2}$ & $\mathbf{- 1 4 2 0 2}$ & -14173 & $\mathbf{- 1 4 1 1 3}$ & $\mathbf{- 1 4 0 1 3}$ & $\mathbf{- 1 3 9 5 4}$ & $\mathbf{- 1 3 8 6 4}$ & -13646 \\
-3.0 & -14700 & $\mathbf{- 1 4 3 0 5}$ & $\mathbf{- 1 4 2 9 0}$ & $\mathbf{- 1 4 2 7 6}$ & $\mathbf{- 1 4 2 5 7}$ & -14227 & $\mathbf{- 1 4 1 6 7}$ & $\mathbf{- 1 4 0 6 5}$ & $\mathbf{- 1 4 0 0 4}$ & $\mathbf{- 1 3 9 1 1}$ & -13692 \\
$(6070)$ & -14706 & -14309 & -14294 & -14280 & -14261 & -14231 & -14170 & -14069 & -14009 & -13917 & -13698 \\
+3.0 & -14714 & -14317 & -14304 & -14289 & -14270 & -14240 & -14179 & -14079 & -14017 & -13925 & -13706 \\
+7.5 & -14759 & -14360 & -14345 & -14331 & -14310 & -14282 & -14220 & -14118 & -14057 & -13964 & -13742 \\
+15.0 & -14940 & -14517 & -14501 & -14486 & -14467 & -14438 & -14375 & -14271 & -14203 & -14103 & -13871 \\
\hline
\end{tabular}

could cause a change in the mean motion of an asteroid pair component by at least $0.010^{\prime \prime} \mathrm{yr}^{-1}$ backward to $t$, were selected in Table 3 and listed by numbers next to asteroid pairs (for perturbers common in all databases) or below them (for perturbers found only in particular database). Perturbers are roughly ordered according to the computed change in the mean motion they cause and also according to the number of encounters with the pair component (that changes $>0.010^{\prime \prime} \mathrm{yr}^{-1}$ in its mean motion). By far the strongest main belt perturbers for most of the pair components we studied are (1) Ceres and (4) Vesta. Not only did they change the mean motion of several asteroids by more than $0.100^{\prime \prime} \mathrm{yr}^{-1}$, but they also perturbed them repeatedly. Only the components of pair (88259) $2001 \mathrm{HJ}_{7}-1999 \mathrm{VA}_{117}$ located in the Hungaria region were relatively unaffected by them; instead, their strongest perturber was (2) Pallas. There are some small perturbers that are listed only for a particular database. They encountered a given asteroid only once and their strong perturbing effect was caused primarily by their short minimum nominal encounter distance. Owing to the large uncertainty in computed distances, it is possible that their effect could, in fact, be weaker. This is why computations with different databases provide different lists and different orders of strong perturbers, except for the few strongest ones. The effect of uncertainty in the mass of smaller perturbers is not as important as the effect of uncertainty in distance. In fact, the weak effect of uncertainty in mass was noticed in the similarity of close approach dates back to $\approx 30 \mathrm{kyr}$ when integrations using only CPV (except for the planetary perturbers) were performed with two different JPL ephemerides, in which the masses of CPV differed slightly between ephemerides. Table 2 contains mixed results from both ephemerides, so the approximate dates of older approaches cover slightly wider time intervals than dates obtained from just one particular ephemeris in Table 3.

For integrations with gradual shifts in the semimajor axis, only nominal dates of encounters between components are listed in Table $3 ; t_{\mathrm{p}}$ and $t_{\mathrm{r}}$ denote dates for prograde and retrograde rotators, respectively. Minimum encounter velocities and distances, as well as the strong perturbers acting on them are comparable to those listed for the integrations without shifts. Pairs from Table 2 that are not displayed in Table 3 seem to be older than $43 \mathrm{kyr}$, because components of those pairs approached each other with minimum encounter velocities $>2.0 \mathrm{~m} \mathrm{~s}^{-1}$ (higher than escape velocity) or their minimum nominal distances were too large, or both. For example, asteroids (99052) $2001 \mathrm{ET}_{15}$ and (291788) $2006 \mathrm{KM}_{53}$ approached each other with an encounter velocity of only $1.1-1.5 \mathrm{~m} \mathrm{~s}^{-1}$, but as the encounter happened relatively recently $(\approx 5.2-5.3 \mathrm{kyr}$ ago $)$, it is expected that nominal minimum distances (of several thousand $\mathrm{km}$ ) have relatively small uncertainties, so that no possible clone would enter the
Hill's radius, which is within $1000 \mathrm{~km}$. $\left(R_{\text {Hill }}<1000 \mathrm{~km}\right.$ for most of the other presented pairs.) Moreover, the times of minimum distances between those components did not coincide with the times of the lowest encounter velocity.

Six out of nine pairs in Table 3 have relative encounter velocities within $1.0 \mathrm{~m} \mathrm{~s}^{-1}$. Although nominal minimum distances usually exceed the Hill's radius by a few times, it is possible that the distance uncertainties are such that the Hill's radius could be reached, and the computed date of closest approach may be very close to the date of pair formation.

(6070) Rheinland - (54827) $2001 \mathrm{NQ}_{8}$. Vokrouhlický et al. (2011) reveals a retrograde rotation of (6070) Rheinland and estimates the age of the pair to be $17.2 \pm 0.2 \mathrm{kyr}$ assuming retrograde rotation of the smaller component (54827) $2001 \mathrm{NQ}_{8}$, or $16.75 \pm 0.15 \mathrm{kyr}$ supposing its prograde rotation. They use clones with drifts in semimajor axis from the interval $-7.95-0 \mathrm{~m} \mathrm{yr}^{-1}$ for the larger component and $-15.0-+15.0 \mathrm{~m} \mathrm{yr}^{-1}$ for the smaller one, respectively. According to Table 3, age estimation including the Yarkovsky effect seems to be about $16.3 \pm 0.1 \mathrm{kyr}$ for retrograde rotations. It is younger by about $0.9 \mathrm{kyr}$ than the previous estimate. The main belt perturbers seem to be responsible for the difference.

A bit more detailed view on the sensitivity of the Yarkovsky effect to age estimation can be seen in Table 4, which contains the nominal dates of approaches between a few Yarkovsky clones computed with 262 main belt perturbers using the F orbital element database (and JPL DE406 ephemeris). Again, clones with negative numbers represent retrograde rotators, while those with positive numbers represent prograde rotators (units are in $\mathrm{m} \mathrm{yr}^{-1}$ ). For the expected range of semimajor axis drifts according to Vokrouhlický et al. (2011), the dates of encounters are emphasized in boldface. If the smaller component is a prograde rotator, the age estimation would be about $16.0 \pm 0.2 \mathrm{kyr}$. It is younger than the previously published estimate by about $0.7-0.8 \mathrm{kyr}$. Similar tables (with slightly shifted values) can be constructed using other databases (and with JPL DE421 ephemeris) apparently confirming the result mentioned with uncertainties. The shift is within a few years. For example, for components drifts $\Delta a / \Delta t=-7.5$ and $+15.0 \mathrm{~m} \mathrm{yr}^{-1}$, respectively, the nominal date of encounter would occur between -13851 and -13834 depending on the database used.

Although the integrator SOLEX (its used and available version) does not allow users to include a fictitious transverse acceleration into computations yet, no serious contribution to age estimation uncertainty should come from the periodic adjustment of the semimajor axis of each member of pairs by a fixed amount. For example, if just the $1000 \mathrm{yr}$-long step (and corresponding adjustment of the semimajor axis) is used instead of the $2000 \mathrm{yr}$ one in Table $3, t_{\mathrm{p}}$ and $t_{\mathrm{r}}$ values using the $\mathrm{F}$ database would be 
Table 5. Comparison of orbital elements for asteroid (21) Lutetia.

\begin{tabular}{lllllll}
\hline \hline ELM & $a$ & $e$ & $i$ & $\omega$ & $\Omega$ & $M$ \\
& {$[\mathrm{AU}]$} & & ${ }^{\circ}{ }^{\circ}$ & $\begin{array}{l}\omega \\
{\left[{ }^{\circ}\right]}\end{array}$ & $\begin{array}{l}\left.{ }^{\circ}\right] \\
{\left[{ }^{\circ}\right]}\end{array}$ \\
\hline $\mathrm{A}$ & 2.4354630548 & 0.16293787077 & 3.0640286042 & 250.36839633 & 80.894091686 & 29.481246404 \\
$\mathrm{~B}$ & 2.43546558 & 0.16293836 & 3.064055 & 250.368155 & 80.894069 & 29.480922 \\
$\mathrm{~F}$ & 2.4354655771 & 0.162938419 & 3.0640574 & 250.3682494 & 80.8939893 & 29.4809160 \\
$\mathrm{~J}$ & 2.4354656 & 0.16293843 & 3.06405 & 250.36793 & 80.89430 & 29.4809145 \\
$\mathrm{M}$ & 2.4354656 & 0.1629385 & 3.06405 & 250.36793 & 80.89426 & 29.48095 \\
\hline
\end{tabular}

-13963 (instead of -13964 ) and -14218 (instead of -14220), respectively.

The initial orbital uncertainty for this pair should also not enhance the age estimation uncertainty. In fact, initial orbital elements of several used perturbers exceeded their uncertainty limits. One source of uncertainty comes from rounding, because the number of decimal places in some databases was too low. The second source of uncertainty is startling. After orbital evolution was finished, it was found that initial orbital elements of a few perturbers differed by much more than expected from estimated errors. The most striking example is mentioned in Table 5 with orbital elements of the asteroid (21) Lutetia extracted from all five databases for the same epoch of JD 2456000.0 (Mar. 14, 2012). Although the uncertainty of the semimajor axis should be on the order of $4 \times 10^{-9} \mathrm{AU}$ as stated in the $\mathrm{A}$ and $\mathrm{J}$ databases, it differed much more in the A database ${ }^{6}$. Even though the encounter between components using the A database also occurred close to the date computed using other databases. For our rough age re-estimation, no additional detailed analysis is therefore needed for this pair.

(88259) $2001 \mathrm{HJ}_{7}-1999 \mathrm{VA}_{117}$. The close approach between components located in the Hungaria region was previously reported by Milani et al. (2010) and by Pravec et al. (2010). It occurred $\approx 34 \mathrm{kyr}$ ago according to the former. The latter take into account the Yarkovsky effect as well as planetary perturbations, and prefer the age of $60_{-15}^{+50} \mathrm{kyr}$. If that were the case, the pair would have formed during the previous encounter of its components, when their minimum distance seemed to be less than the Hill's radius. Indeed, according to trial computations including the integrations that involved the Yarkovsky effect (with even different semimajor axis drifts than shown in Table 3), the nominal minimum distance between components for all five databases was always confined to $2-3 \times 10^{3} \mathrm{~km}$, which is slightly above the Hill's radius. Even after resolving the sense of rotation of the components, it may still be necessary to model the mutual perturbation of components in order to resolve which solution for the age is true. As for the effect of main belt perturbers, it was mentioned above that the strongest perturber was (2) Pallas, but it was not the only culprit responsible for the difference $>1 \mathrm{kyr}$ between the date of closest approach reported by Milani et al. and the dates found in Table 3, because according to Table 2 the difference should be within $0.3 \mathrm{kyr}$. The difference in initial orbital elements (due to added astrometric observations for the first component) used likely plays a significant role.

(111962) $2002 \mathrm{GP}_{75}$ - (280008) $2001 \mathrm{UR}_{224}$. In several integrations incorporating semimajor axis drift, the minimum nominal distance and relative encounter velocity were even less than shown in Table 3, enhancing the probability that the pair could

\footnotetext{
6 This inconsistency could also be seen in older versions of the A database, but apparently, it has been fixed in more recent version.
}

have formed within the period under study. In computations with just CPV, encounters happened more recently by a few (2-6) hundred years when the JPL DE421 ephemeris was used instead of DE406 for each database. However, age uncertainty is dominated by the Yarkovsky effect. Taxonomic class being L/S according to SMASS data was recognized for the larger component (111962) $2002 \mathrm{GP}_{75}$ (Willman et al. 2010), but no discussion of the pair's age has been found to date.

(180906) $2005 \mathrm{~KB}_{6}-(217266) 2003 \mathrm{YR}_{67}$. The analysis does not suggest a reliable date of pair formation. For retrograde rotations of the components, especially with the stated semimajor axis drifts, the difference between the mean anomalies of their orbits changed by only a few degrees during the interval studied using initial conditions from four of the databases, indicating the age may greatly exceed $43 \mathrm{kyr}$. By contrast, in one computation for prograde rotations, the nominal minimum distance between components is miniscule, only $33 \mathrm{~km}$, which is well within the Hill's radius. According to trial computations with the enhanced semimajor axis drifts, it was found that encounter might occur quite recently. For example, with drifts $\Delta a / \Delta t=-45.0 \mathrm{~m} \mathrm{yr}^{-1}$ for both components, the nominal date of their encounter would occur as early as in -13712 using the F database, and the encounter velocity in such case would be $0.3 \mathrm{~m} \mathrm{~s}^{-1}$. In computations with just CPV, encounters happened a few thousand years earlier (pair would be older) when the JPL DE421 ephemeris was used instead of DE406 for each database. Since the difference between absolute magnitudes of components is small (in each database $\Delta H \leq 0.3$, while (217266) $2003 \mathrm{YR}_{67}$ is a bit brighter), the pair might have formed from a synchronous binary (Jacobson \& Scheeres 2011). No discussion of the pair's age has been found to date. No wonder - according to a few clones mentioned here, it is apparent that the uncertainty in pair age is currently huge. This pair can be the youngest one, as well as the oldest one, from presented pairs.

(229401) 2005 SU $_{152}-2005$ UY $_{97}$. Pravec et al. (2010) report an age of $17_{-10}^{+25} \mathrm{kyr}$ for the pair. All results in Table 3 are well within that wide interval. The effect of main belt perturbers could not be seen until the uncertainty caused by the Yarkovsky effect is heavily reduced.

$2005 \mathrm{GS}_{180}-2008 \mathrm{FK}_{107}$. Like many other young pairs even this one was revealed earlier (Rożek et al. 2011), though without any discussion of its age. The orbital elements are derived from a small number of astrometric observations (28 and 21), so further observations are desirable to reduce initial uncertainties. Large telescopes would be needed to provide photometric observations, because objects are rather faint. Moreover, even the drift in semimajor axis can be much larger than presented in Table 3, so the age estimation has a larger uncertainty than indicated. 
It is less clear if the three remaining pairs in Table 3, with slightly higher encounter velocity and longer nominal minimum distances between components, could also be formed within the studied interval, or if they were formed in some of the previous mutual encounter of components. The age of one of these pairs, (5026) Martes - $2005 \mathrm{WW}_{113}$, is $17 \pm 2 \mathrm{kyr}$ according to Pravec et al. (2010). The current analysis shows that the encounter velocities range widely. Although there are some slow approaches between components within $1.0 \mathrm{~m} \mathrm{~s}^{-1}$, all those nominal distances are too large. It must also be mentioned that the most recent close encounter between components occurred just $\approx 2.5$ kyr ago, though with a bit higher encounter velocity. Because there are three slow encounters within the period studied, mutual perturbation of components should probably be considered for the age estimation. Mutual perturbations should also be taken into account for the age estimation of the pair (224801) $2006 \mathrm{UQ}_{127}-2008 \mathrm{SZ}_{124}$, because its members might encounter twice during the period studied, as can be seen in Table 2, and the relative velocities are similar in both encounters. As there are some slow approaches within $1.0 \mathrm{~m} \mathrm{~s}^{-1}$ at the older encounter, it is mentioned in Table 3. No information about the pair has been found to date. In this case, the components seem to be of similar size. Members of the remaining pair, (80218) $1999 \mathrm{VO}_{123}$ and (213471) $2002 \mathrm{ES}_{90}$, also seem to be of similar size. As no nominal encounter velocity is within $1.0 \mathrm{~m} \mathrm{~s}^{-1}$ and all nominal minimum distance exceed $10 \times 10^{3} \mathrm{~km}$, this pair probably formed in one of the previous encounters between components. The expected smaller orbit uncertainties at the recent encounter make it less likely that the minimum distance could reach the Hill's sphere.

Even though the effect of main belters on asteroid-pair age estimation is usually buried in huge age uncertainties caused by the Yarkovsky effect, in principle, it can be assessed from the comparison of dates of closest approaches between pair components computed with different number of perturbers as was shown in Tables 2 and 3. Interestingly, nominal dates of encounters using the $\mathrm{J}$ and $\mathrm{M}$ databases, respectively, provide results of two geometrical clones of 259 perturbers other than CPV, because corresponding $t_{\mathrm{CPV}}$ values are exactly the same in Table 3.

\section{Conclusion}

The results of this work are preliminary, but do suggest how to further refine age estimation of several young asteroid pairs in the future. The influence of main belt perturbers on asteroid-pair age estimation is usually not as important as the Yarkovsky effect. But, in cases with reduced initial uncertainties, i.e. after both the sense of rotation/pole position, and absolute magnitudes/sizes are known more precisely, the perturbations by the largest main belters, especially (1) Ceres and (4) Vesta, have a noticeable effect. Currently, the effect of main belters is not negligible only for estimating the age of the pair (6070) Rheinland - (54827) $2001 \mathrm{NQ}_{8}$.

Computations leading to precise age determination would be more reliable if a number of clones of several possible orbits were used, if perturbations by pair components were taken into account, if higher precision was chosen in the integrator and if results from other integrators were used for comparison. That can be the aim of one's future work, but in general, the age determination for any pair using many main belt perturbers is too complex. Except for modeling the Yarkovsky effect, which is usually the most important, one also needs a reliable source for

- orbital elements with uncertainties not only for pair components, but also for perturbers,

- masses with uncertainties for all the perturbers used,

- planetary ephemerides covering several millennia.

However, no integrator could probably cope with geometrical clones or with "mass" clones of perturbers (currently, masses for many main belt perturbers are just roughly estimated) needed for that task, not to mention concern about its worthiness. It may happen that observers obtain valuable additional astrometric and/or photometric observations that enable age uncertainty to be heavily reduced before some analyzer finishes extensive modeling of all possible uncertainties.

Acknowledgements. I am indebted to Prof. A. Vitagliano for providing both the updated version of SOLEX package and his kind advice. Thanks also go to all other authors of databases and freewares that were used in this work, namely E. Bowell (Astorb), G. V. Williams and T. Spahr (MPCOrb), A. Milani, Z. Knežević and B. Novaković (AstDys), JPL team (JPL database) and B. J. Gray (for the FIND ORB package), as well as to the reviewer D. Vokrouhlický for fruitful discussions during the review process. Special thanks go to W. Zielenbach and J. Adams for many suggestions on the readability of the text and kind language corrections. This work was supported by the project RVO:67985815, Grant Agency of the Czech Republic, Grant number P209/12/0229.

\section{References}

Baer, J., Chesley, S. R., \& Matson, R. D. 2011, AJ, 141, 143

Bottke, W. F., Jr., Vokrouhlický, D., Rubincam, D. P., \& Brož, M. 2002, in Asteroids III, eds. W. F. Bottke Jr., A. Cellino, P. Paolicchi, \& R. P. Binzel, (Tucson: University of Arizona Press), 395

Bottke, W. F., Jr., Vokrouhlický, D., Rubincam, D. P., \& Nesvorný, D. 2006, Ann. Rev. Earth Planet. Sci., 34, 157

Chesley, S. R., Ostro, S. J., Vokrouhlický, D., et al. 2003, Science, 302, 1739

Duddy, S. R., Lowry, S. C., Wolters, S. D., et al. 2012, A\&A, 539, A36

Jacobson, S. A., \& Scheeres, D. J. 2011, Icarus, 214, 161

Laskar, J., Gastineau, M., Delisle, J.-B., Farrés, A., \& Fienga, A. 2011, A\&A, 532, L4

Milani, A., Knežević, Z., Novaković, B., \& Cellino, A. 2010, Icarus, 207, 769

Nathues, A. 2010, Icarus, 208, 252

Nesvorný, D., \& Bottke, W. F. 2004, Icarus, 170, 324

Nesvorný, D., \& Vokrouhlický, D. 2006, AJ, 132, 1950

Nesvorný, D., Bottke, W. F., Dones, L., \& Levison, H. 2002, Nature, 417, 720

Nesvorný, D., Bottke, W. F., Levison, H. F., \& Dones, L. 2003, ApJ, 591, 486

Nesvorný, D., Vokrouhlický, D., \& Bottke, W. F. 2006, Science, 312, 1490

Pravec, P., \& Vokrouhlický, D. 2009, Icarus, 204, 580

Pravec, P., Vokrouhlický, D., Polishook, D., et al. 2010, Nature, 466, 1085

Rożek, A., Breiter, S., \& Jopek, T. J. 2011, MNRAS, 412, 987

Tedesco, E. F., Noah, P. V., Noah, M., \& Price, S. D. 2002, AJ, 123, 1056

Usui, F., Kuroda, D., Müller, T. G., et al. 2011, Publ. Astron. Soc. Jap., 63, 1117

Vitagliano, A. 1997, Celest. Mech. Dyn. Astron., 66, 293

Vokrouhlický, D., \& Nesvorný, D. 2008, AJ, 136, 280

Vokrouhlický, D., \& Nesvorný, D. 2009, AJ, 137, 111

Vokrouhlický, D., \& Nesvorný, D. 2011, AJ, 142, 26

Vokrouhlický, D., Chesley, S. R., \& Matson, R. D. 2008, AJ, 135, 2336

Vokrouhlický, D., Ďurech, J., Polishook, D., et al. 2011, AJ, 142, 159

Willman, M., Jedicke, R., Moskovitz, N., et al. 2010, Icarus, 208, 758

Zielenbach, W. 2011, AJ, 142, 120 